$v$.

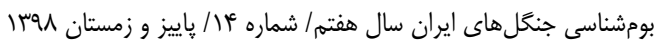

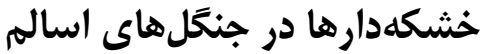

كيومرث سفيدى

دانشيار، دانشكده كشاورزى و منابع طبيعى، داشكاه محقق اردبيلى، اردبيل، ايران، (نويسنده مسوول: Kiomarssefidi@gmail.com)

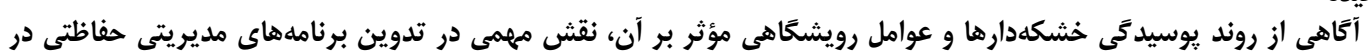

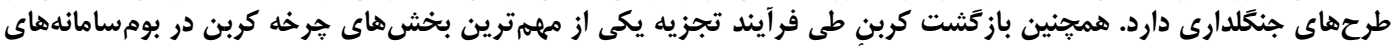

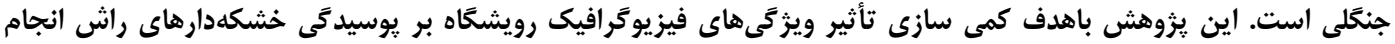

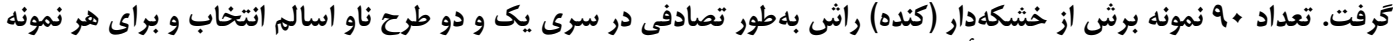

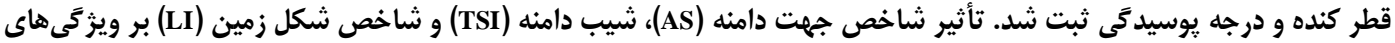

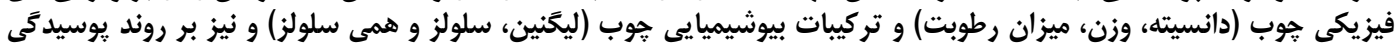

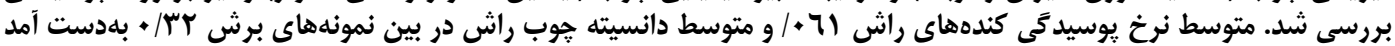

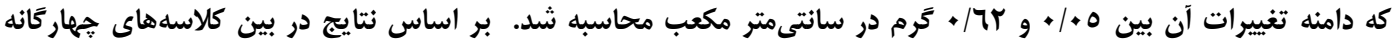

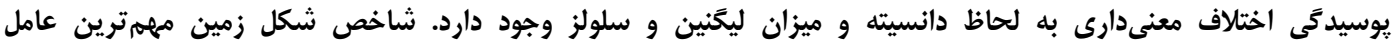

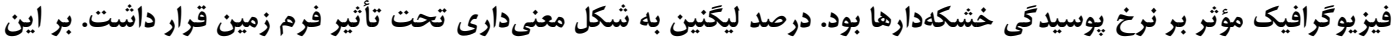

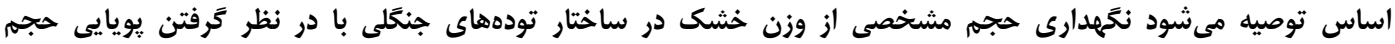

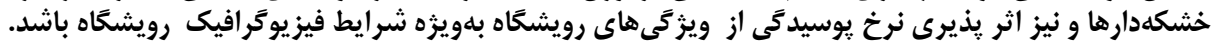

وازههاى كليدى: فيزيوكر افى، نرخ بوسيدَّى، درجه يوسيدكى، شاخص شكل زمين، شاخص شكل شيب دامنه

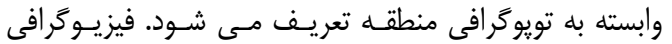

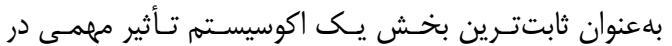

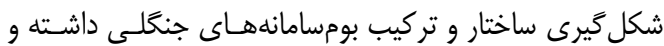

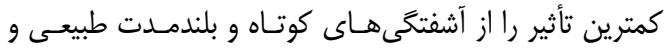

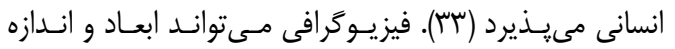

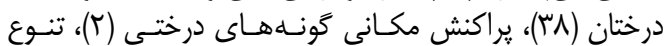

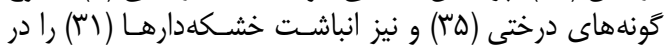

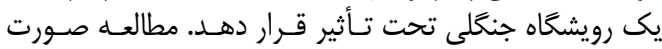

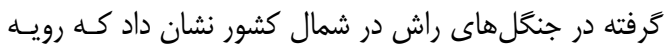

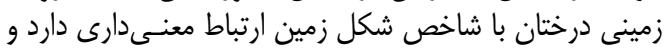

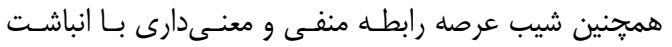

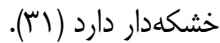

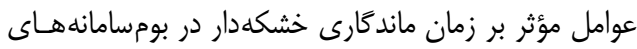

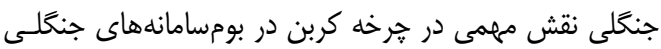

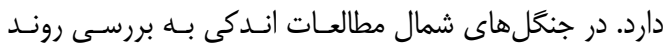

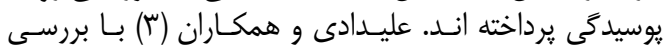

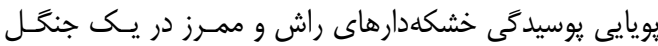

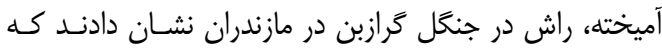

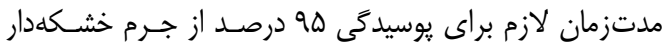

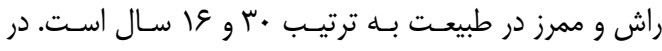

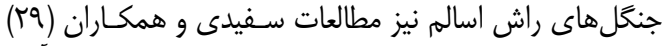

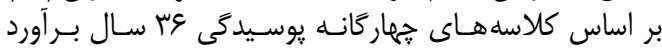

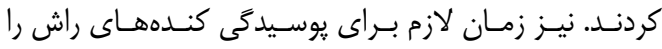
و و همكاران (Tetrillo
مقدمه

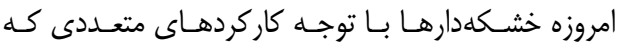

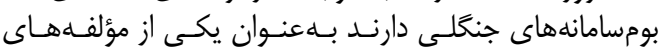

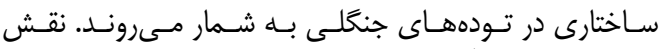

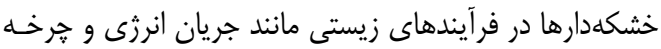

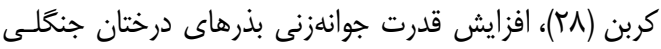

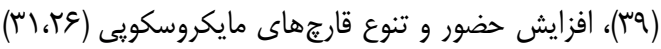

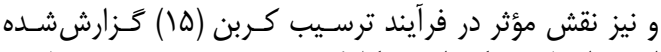

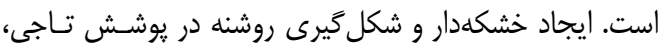

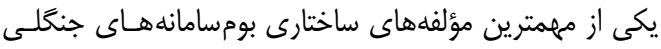

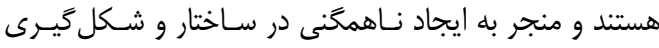

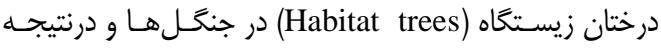

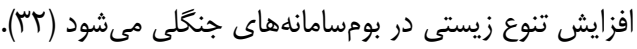

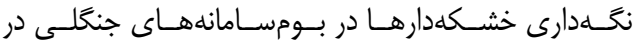

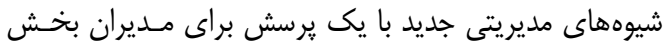

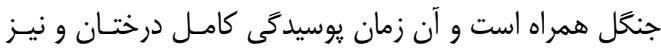

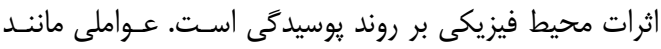

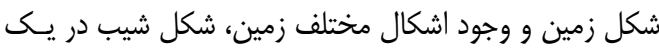

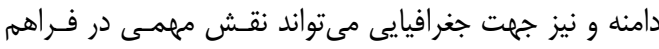

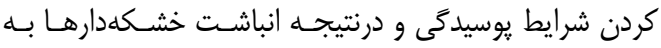

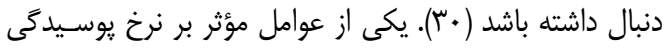

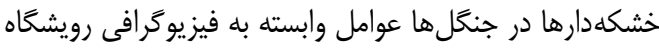

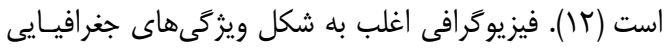

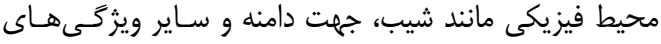




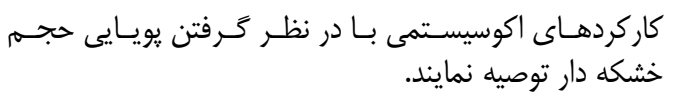

\section{مواد و روشها مورد مطال منطقه مورد مطالعه}

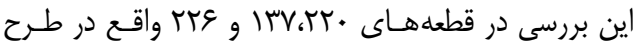

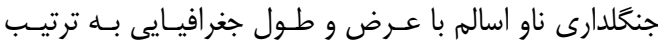

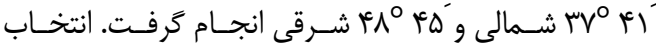

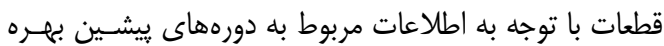

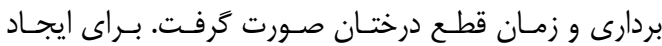

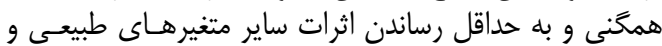

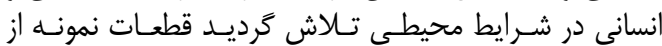

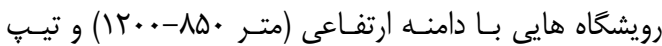

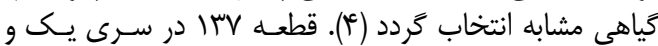

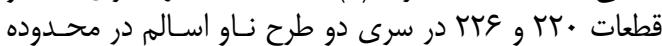

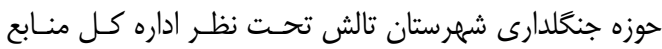

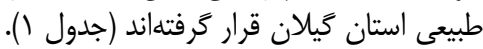

روش جمع آورى دادهن إنها

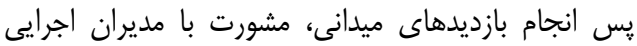

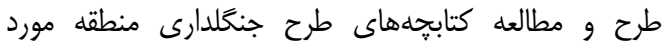

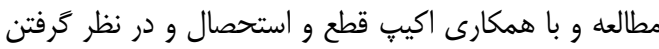

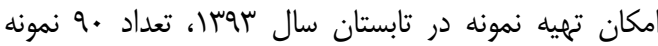

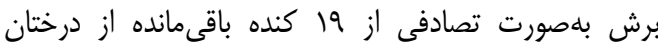

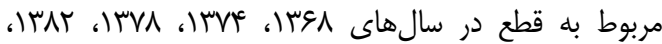

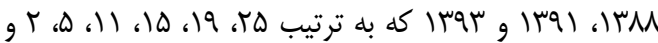

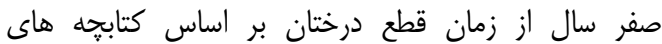

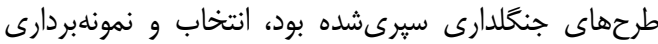

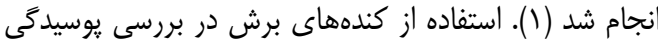

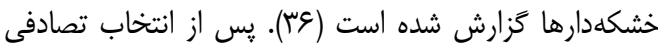

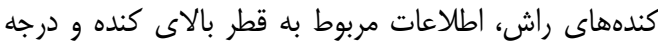

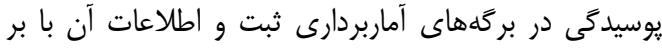

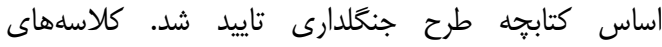

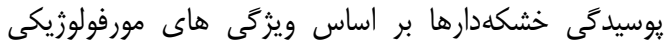

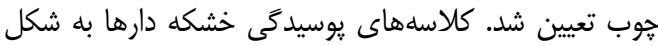

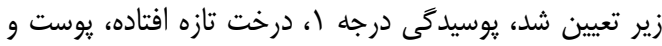

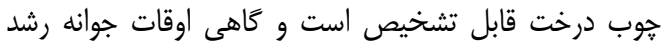

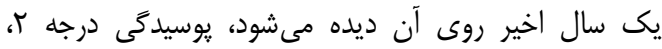

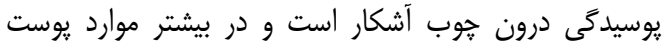

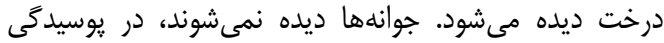

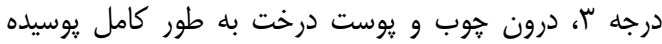

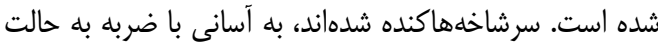

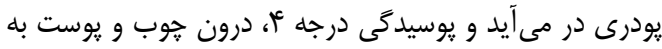

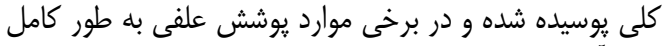
روى آن مستقر شده است (1).

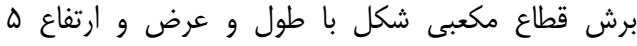

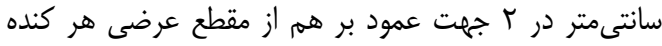

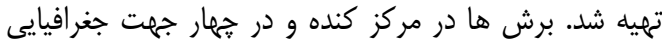

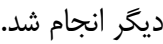

نوئل و لاريكس در ارتفاعات آلٍ در شمال كشور ايتاليا نشـان

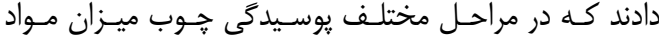

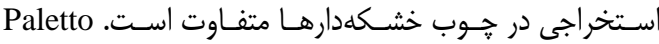

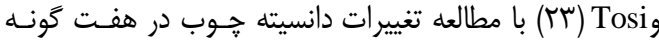

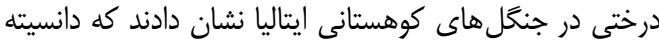

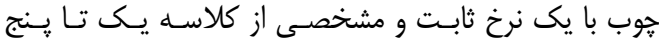

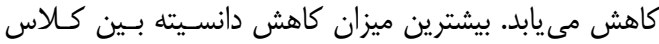

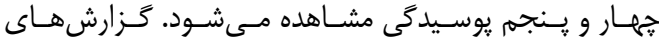

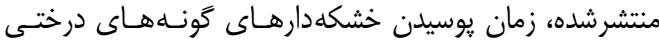

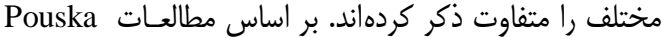

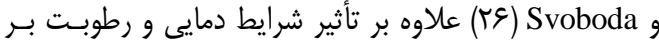

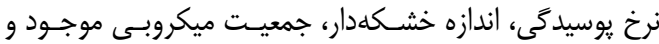

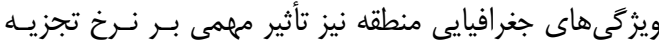

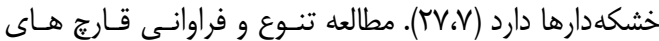

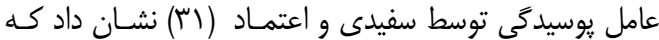

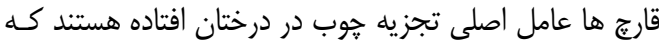

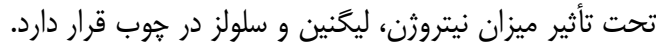

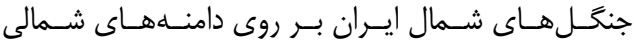

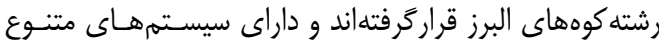

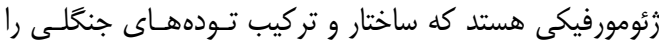

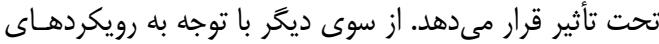

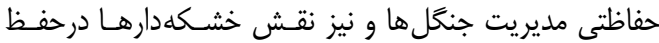

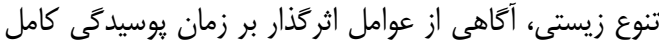

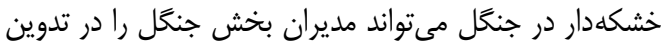

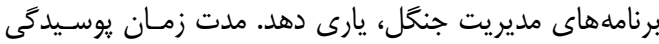

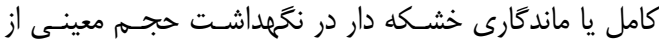

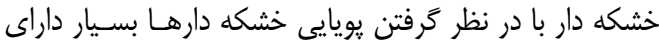

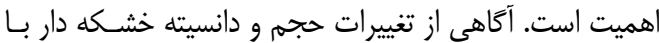

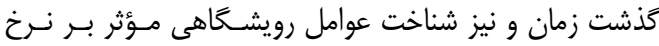

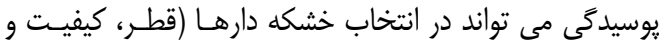

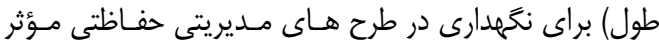

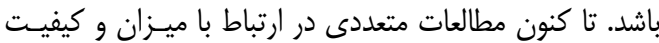

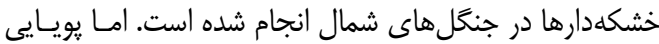

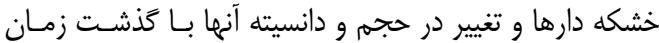

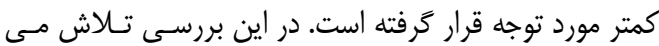

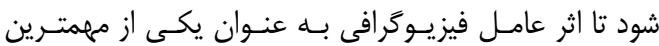

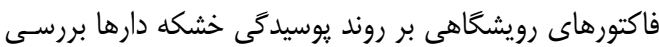

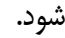
اين يزوهش تلاش دارد تا به اين پيرسش ياسخ دهد كه آيـا

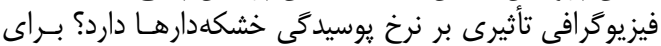

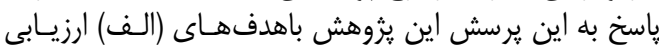

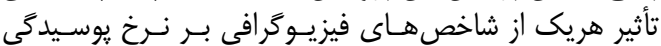

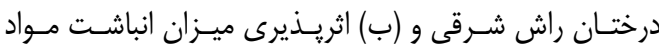

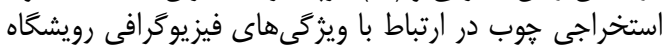

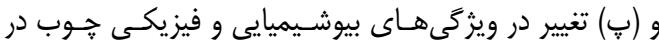

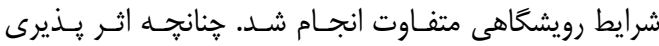

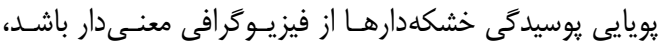

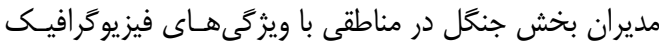

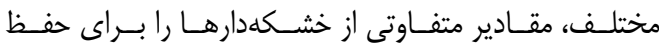


جدول 1- مشخصات كلى قطعات مورد بررسى در جنگلهاى طرح ناو اسالم. Table 1. The general characteristics of the study area in Asalem Forests

\begin{tabular}{|c|c|c|c|c|}
\hline تيب جنگل & يوشش تاجى (\%) & تراكم درختان (اصله در هكتار) & مساحت (هكتار) & قطعه موردبررسى \\
\hline Beech, Maple, Alder & $75-90$ & 331 & 43 & 137 \\
\hline Beech, Hornbeam, Maple & $75-85$ & 320 & 82 & 220 \\
\hline Beech, Hornbeam, Alder & $65-85$ & 288 & 26 & 226 \\
\hline
\end{tabular}

درصد ليخنين نيز به روش كلازون برآورد از رابطه زير محاسبه شد: - ماصنين

(ه) رابطه (ه)

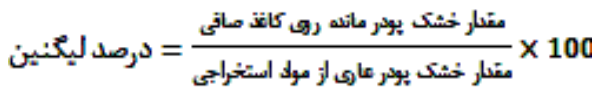

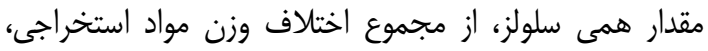

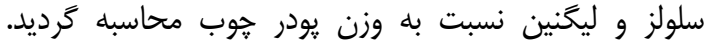

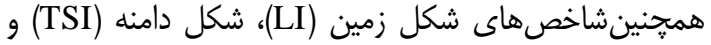

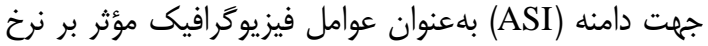

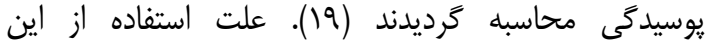
شاخصها بلجاى مشخصههاى توصيفى از شكل زمين مانند

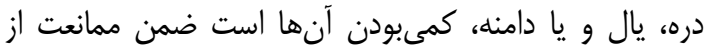

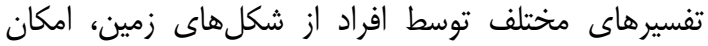
محاسبات أمارى را ميسر ميىسازد.

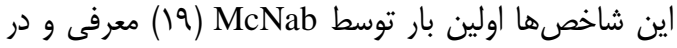

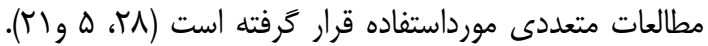

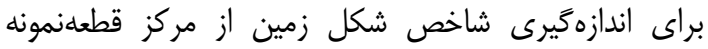

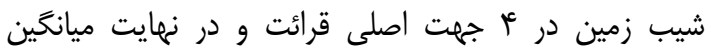

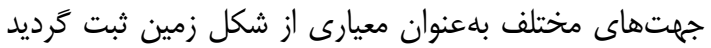

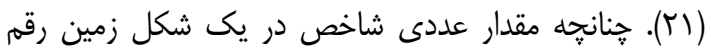

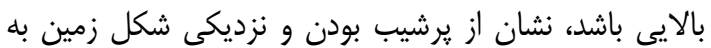

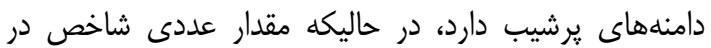

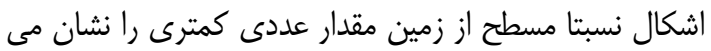

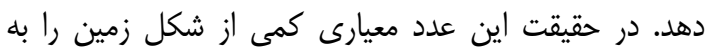

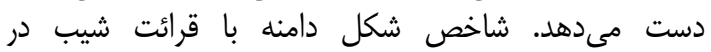

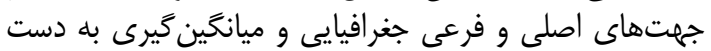

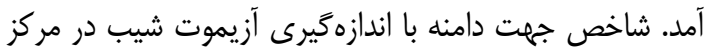

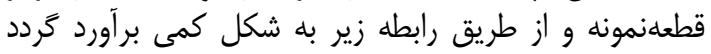

$$
A_{1}=\operatorname{Cos}\left(A_{2}-45\right)+1
$$

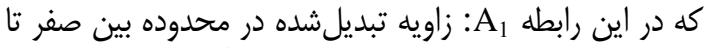

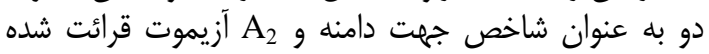

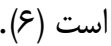

تجزيه و تحليل دادهها

نرمال بودن توزيع دادهها با آزمون كلموگراف اسمهاف اسميرنف و

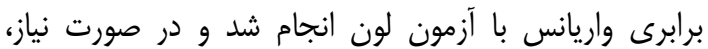

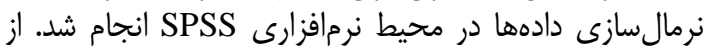

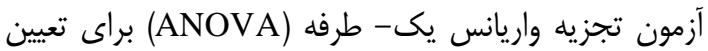

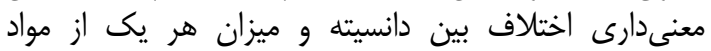

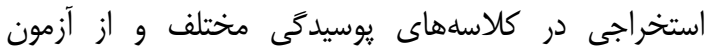

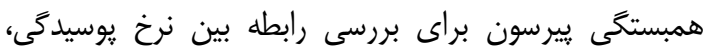

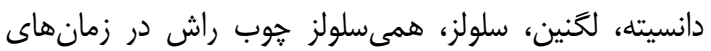

نمونهبردارى از كندههاى جوان با اره موتورى، كندههاى

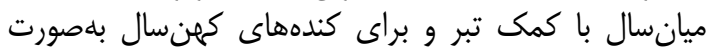

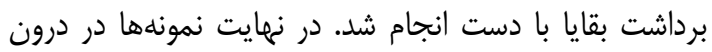

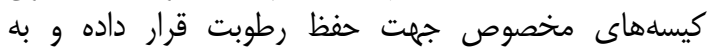

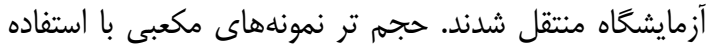

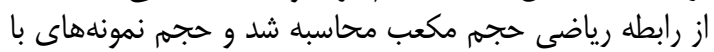

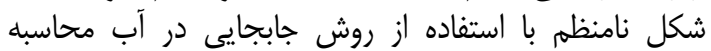

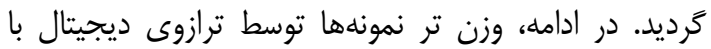

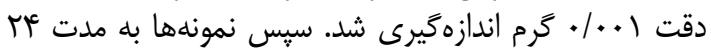

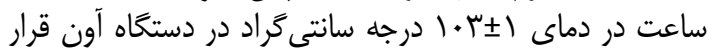

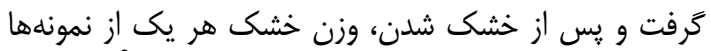

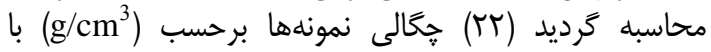
كمك رابطه (1) محاسبه شد:

$$
\rho=m / \mathrm{V}
$$

(1) (1ابطه (1)

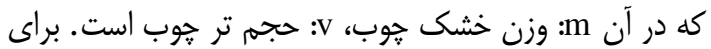

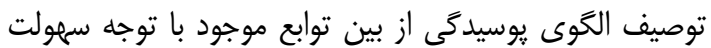

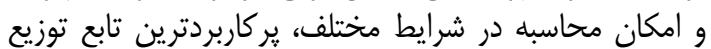

نمايى انتخاب شد (19) - (19).

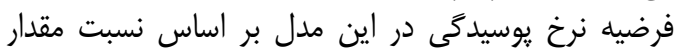

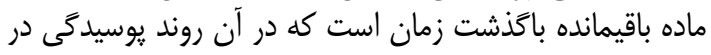

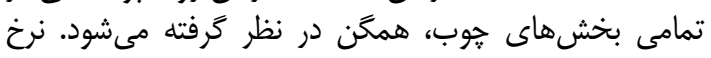

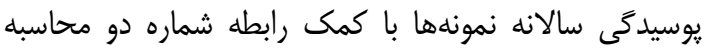
شد) (r) : (r) $k=\frac{\left(\ln D_{0}-\ln D_{t}\right)}{t}$ (r) (1ابطه

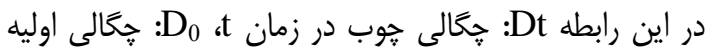

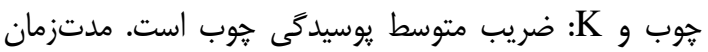

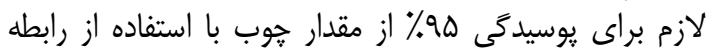
شماره سه محاسبه شد (آم):

$$
T_{95}=-\ln \frac{0.05}{k}=\frac{3}{k}
$$

براى اندازهگيرى تركيب شيميايى خوبها، بر اساس استاندارد

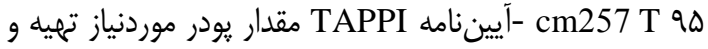
اندازهيرى ليخنين، سلولز، مواد استخراجى محلول دارين در استون

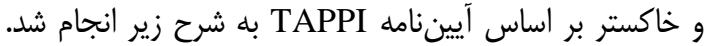

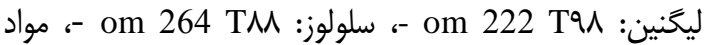
استخراجى محلول در استون: 204 T - cm 9V و خاكستر: - om 211 T. و درصد سلولز از رابطه زير محاسبه گرديد:

(i) رابطه

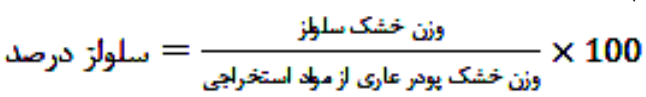


اختلاف معنىدارى بين دانسيته در سالهاى مختلف پِّ از

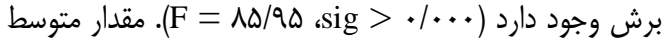

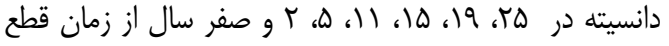

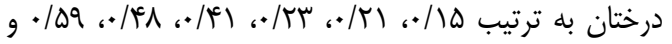

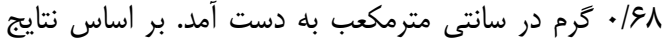

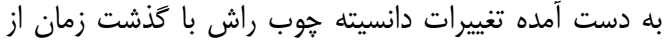
يك تابع نمايى كاهنده بيروى مى كند (شكل (1).
مختلف و هر يك از شاخصهاى فيزيوكرافى شامل شاخص

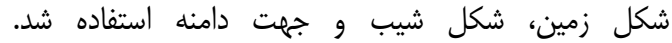

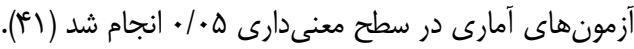

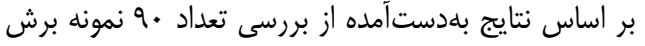

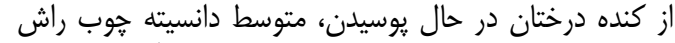

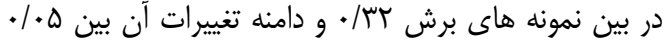

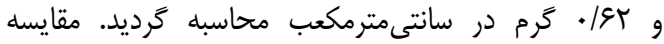

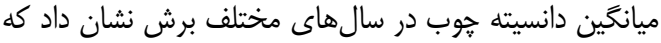

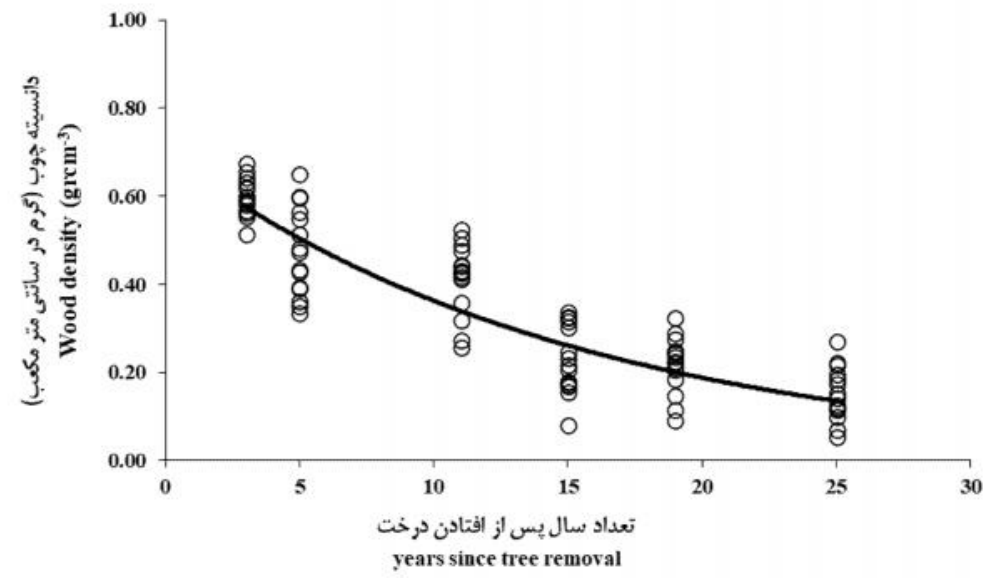

شكل 1- تغييرات دانسيته جوب راش در سالهاى مختلف يس از برش

Figure 1. The change in wood density for the Oriental beech in different years since tree removal

$\mathrm{Dt}=\cdot / 9 \cdot 1 \mathrm{e}^{-\cdot / M V \mathrm{yt}}$

(V) رابطه

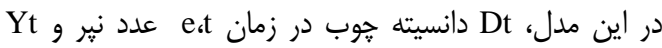

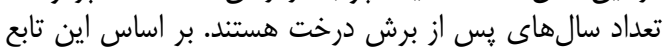

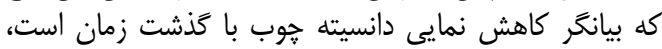

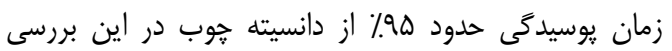

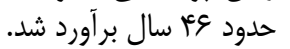

نتايج و بحث

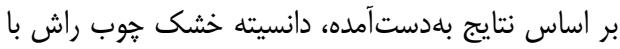

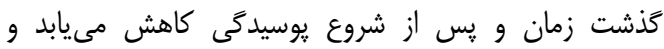

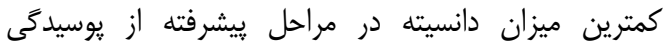

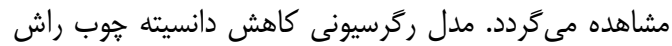

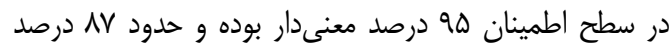

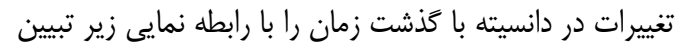

مىنمايد (جدول r).

جدول r- نتايج آناليز واريانس مدل ركرسيونى رابطه بين دانسيته خوب و درجه يوسيدگى در راش (RV) Table 2. Results of ANOVA for the regression model of the relationship between wood density and decay classes for

\begin{tabular}{|c|c|c|c|c|c|}
\hline sig & $\mathrm{F}$ & ميانگين مربعات & مجموع مربعات & درجه آزادى & منبع تغييرات \\
\hline \multirow[t]{3}{*}{$0.0001<$} & 294.357 & 2.117 & 2.11 & 1 & مدل \\
\hline & & & 0.633 & 88 & خطا (باقيمانده) \\
\hline & & & 2.751 & 89 & كل تصحيحنشده \\
\hline
\end{tabular}

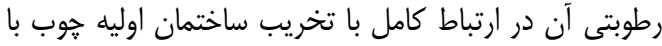

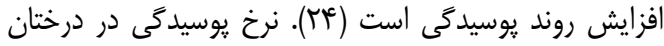

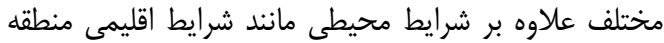

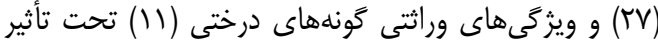

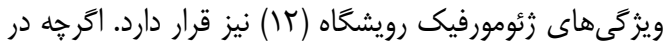

مىتوان بر اساس روند كاهشى موجود، زمان يوسيدگى زانى

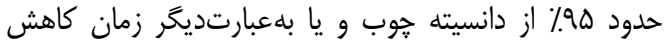

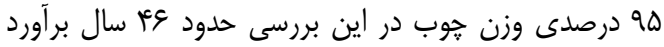

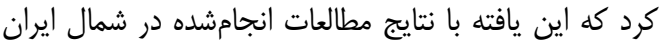

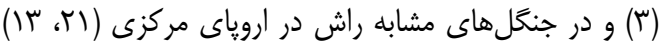

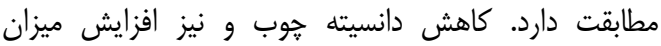




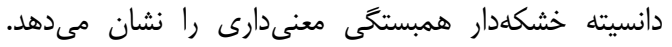

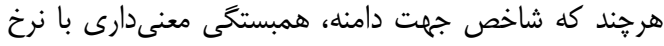

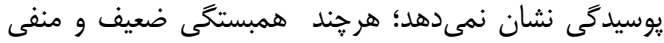

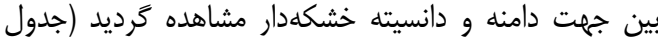

يك مقياس ميانه و كلان تأثير اقليم تأثير جشمخيرى بر روند يوسيدگى دارند (1) (1). بر اساس نتايج اين بررسى شاخص دارئ شكل زمين همبستخى

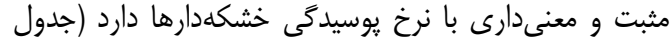

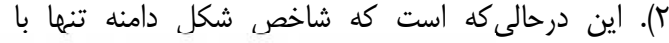

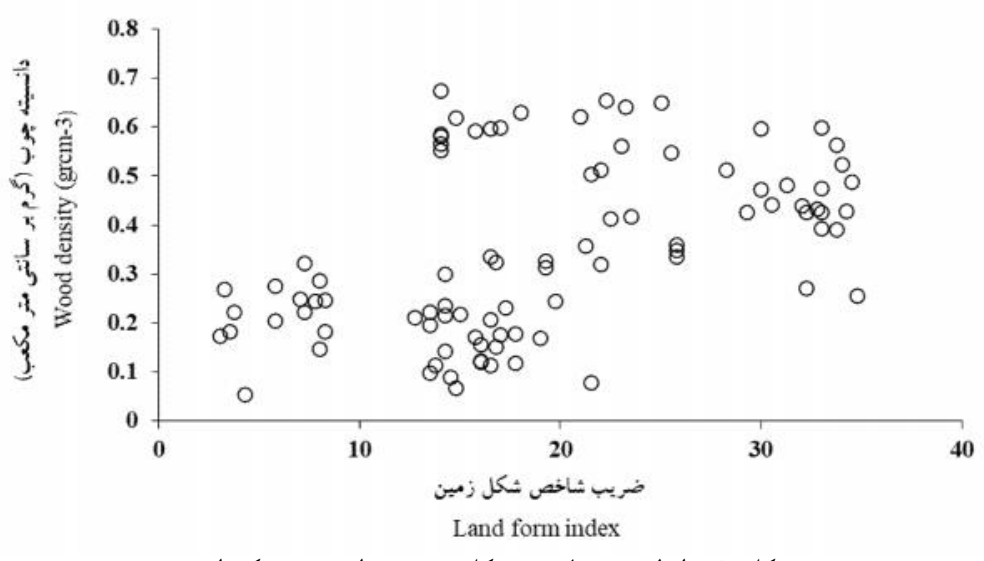

شكل r r- رابطه بين شاخص شكل زمين و دانسيته خشكهدار

Figure 2. The relationship between landform index and dead wood

توجه به كذشت زمان از آغاز فرآيند يوسيدكى جوب مقادير متفاوتى را نشان مى دهن دهند.

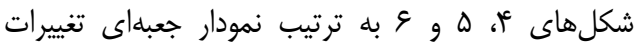

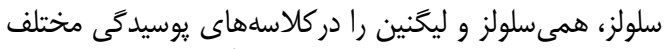

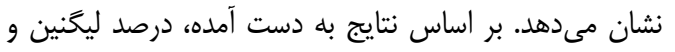

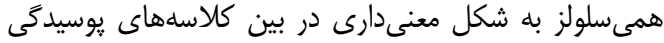

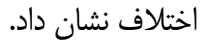

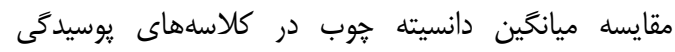

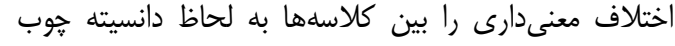

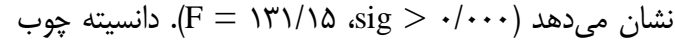

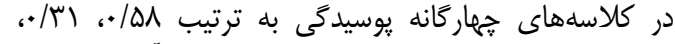

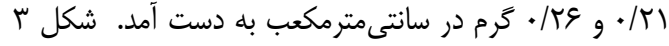

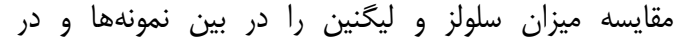

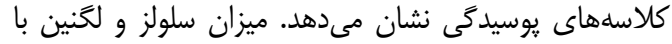

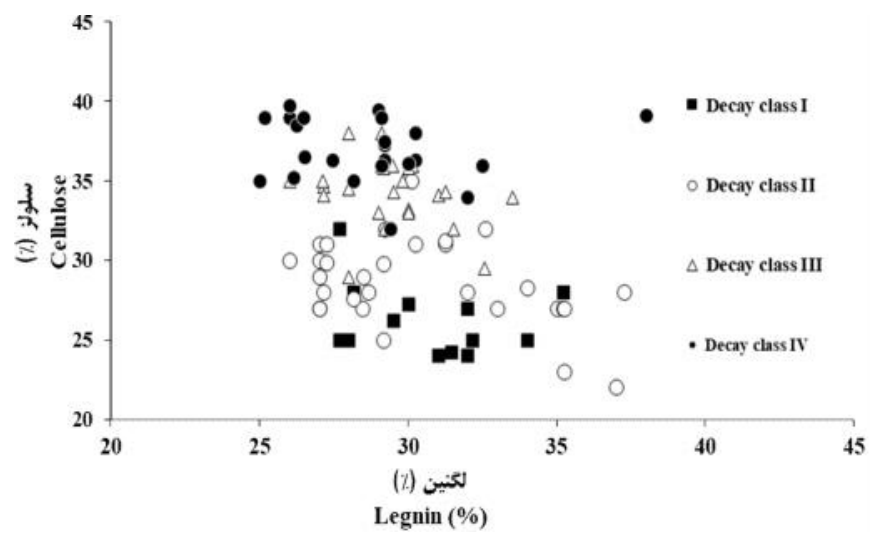

شكل بـ- مقايسه درصد لخنين و سلولز در نمونهها با كلاسه يوسيدگى متفاوت

Figure 3. The comparison lignin and cellulose in different decay classes

زمان ماندكارى خشكهدار متفاوت است. اراضى مى توانند

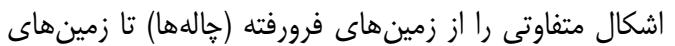

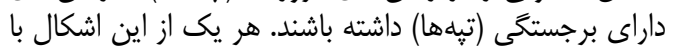

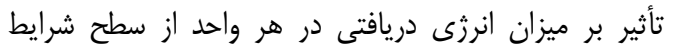

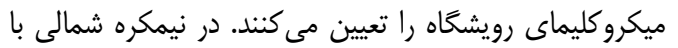
توجه زاويه تابش خورشيدى بيشترين ميزان انرزى در من مناطق دئن
ويثگى هاى فيزيكى هوب (دانسيته، وزن) و و تركيبات

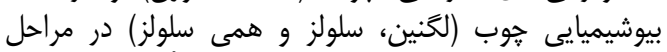

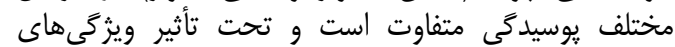

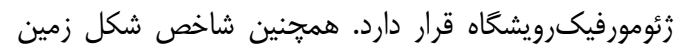

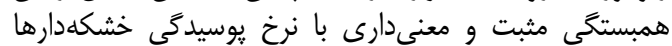

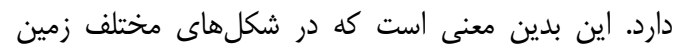




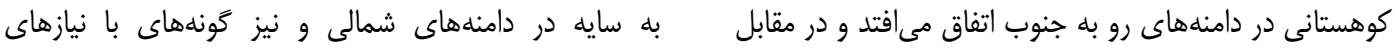

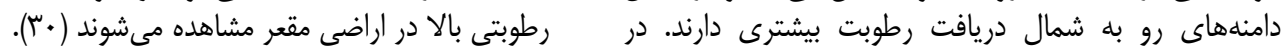

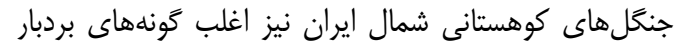

Table 3. Results of correlation test between physiographic indices and decay related wood properties

جدول سـ- آزمون همبستخى بين شاخصهاى فيزيوگر افى و مشخصههاى مرتبط با يوسيدگى خشكهدار شاخص

\begin{tabular}{|c|c|c|c|c|c|c|c|c|}
\hline sig & $r$ & sig & $r$ & sig & $r$ & sig & $r$ & \\
\hline 0.039 & 0.221 & 0.000 & -0.521 & 0.291 & 0.232 & 0.000 & 0.472 & شكل زمين (LI) \\
\hline 0.056 & 0.196 & 0.000 & -0.482 & 0.000 & 0.412 & 0.056 & 0.243 & شكل شيب (TSI) \\
\hline 0.814 & 0.012 & 0.514 & 0.713 & 0.052 & -0.197 & 0.062 & 0.112 & جهت دامنه (ASI) \\
\hline
\end{tabular}

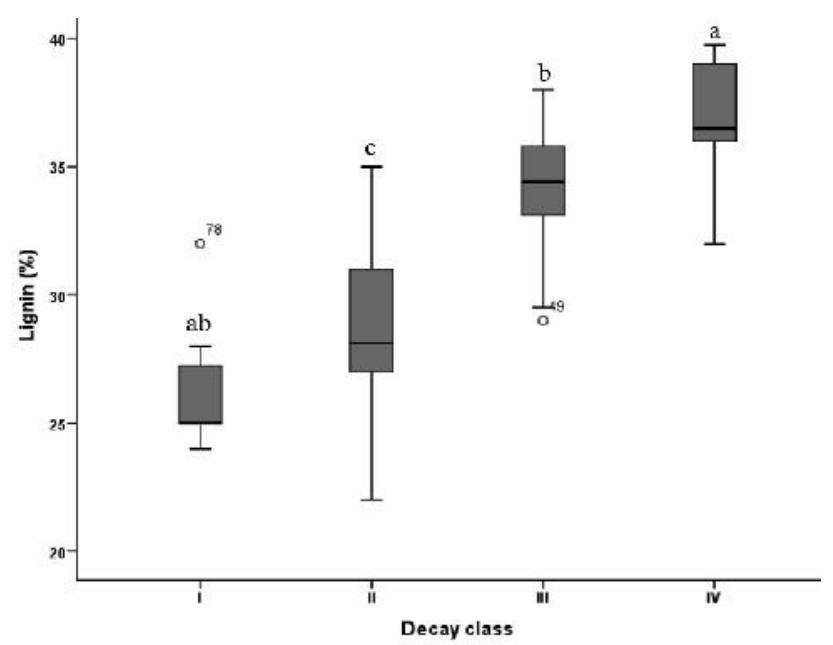

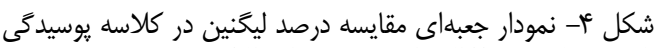

Figure 4. The Boxplot of lignin percent in different decay classes

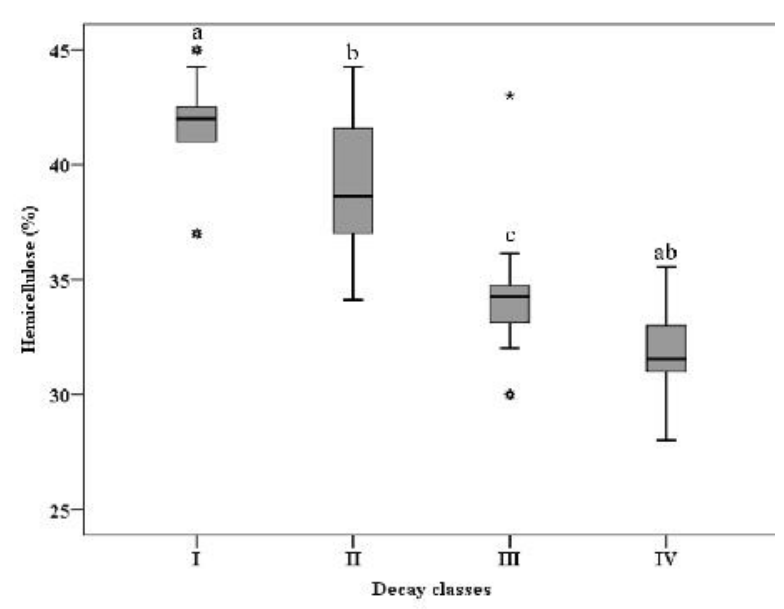

شكل צ- نمودار جعبهاي مقايسه درصد همى سلولز در كلاسه يوسيدگى Figure 6. The Boxplot of hemicellulose percent in different decay classes

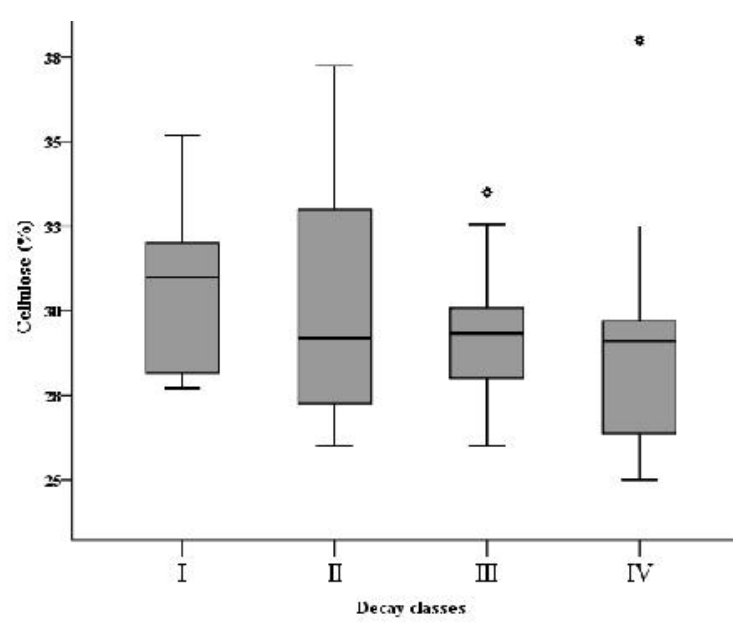

شكل ه- نمودار جعبه اى مقايسه درصد سلولز در كلاسه يوسيدگى Figure 5. The Boxplot of cellulose percent in different decay classes 
(•) (1). در جنكل هاى كوهستانى آينين در كشور ايتاليا براى

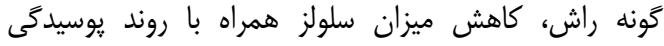

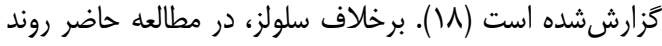

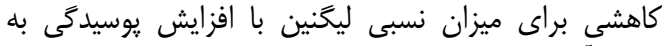

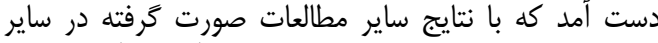

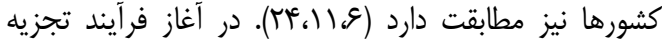

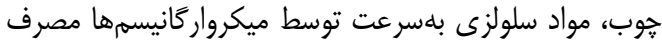

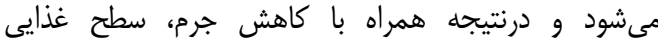

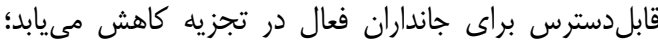

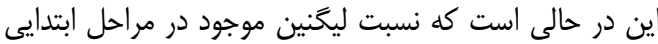

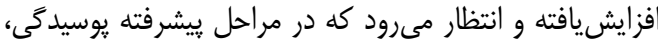

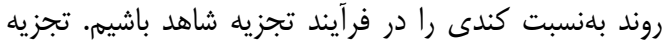

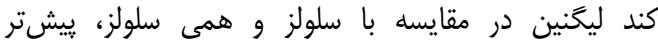

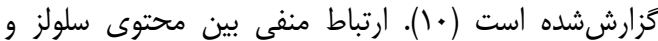

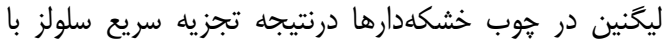

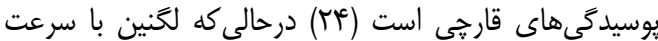

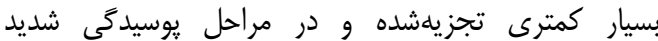

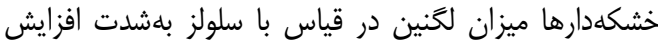

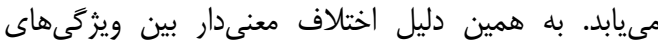

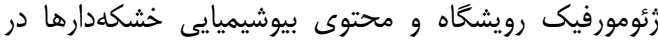

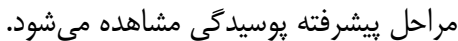

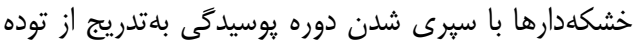

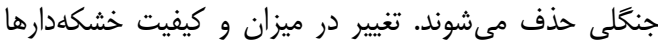

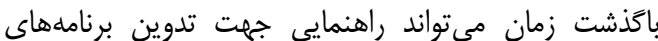

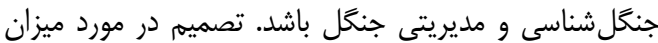

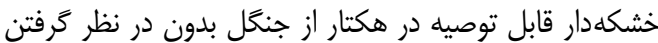

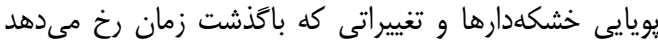

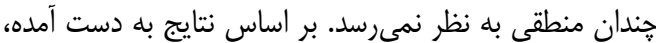

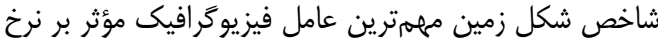

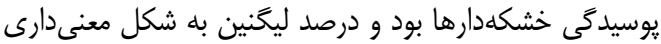

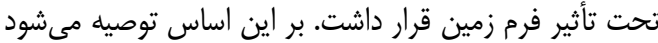

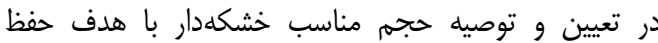

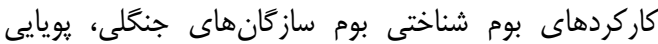

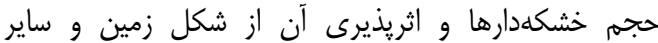

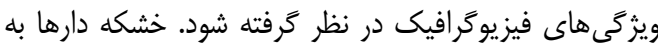

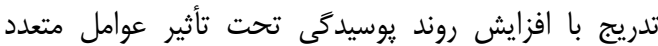

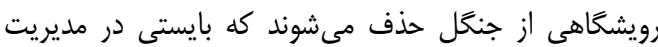

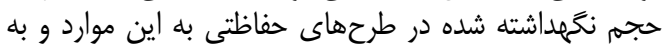

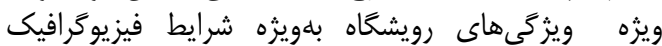
رويشاه توجه كافى شود.

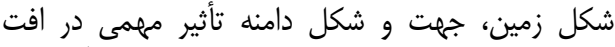

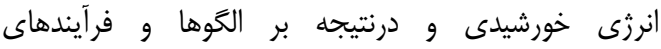

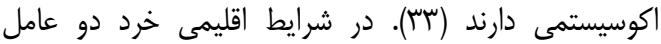

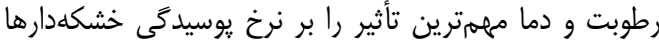

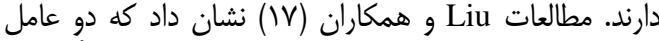

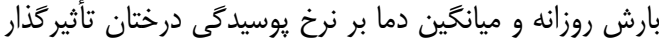

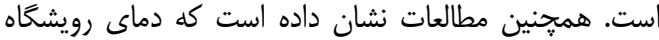

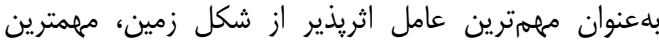

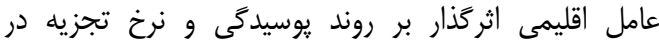

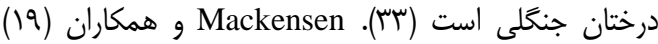

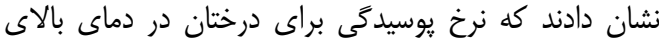

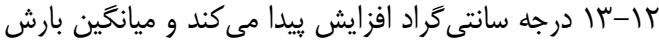

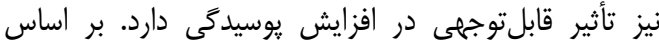

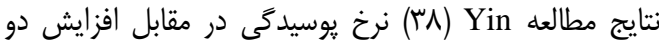

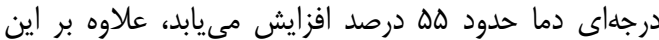

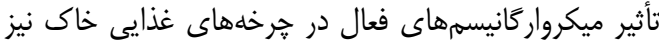

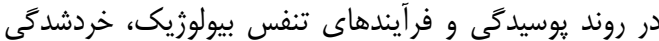

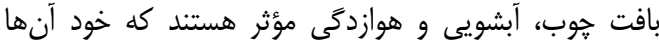

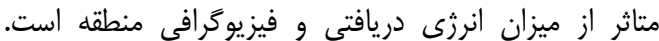

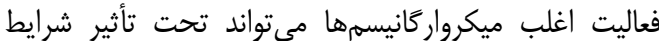

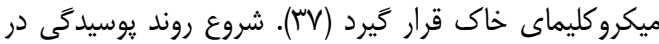

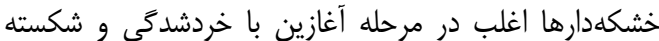

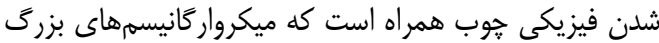

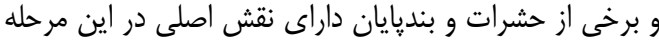

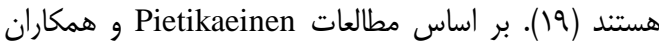

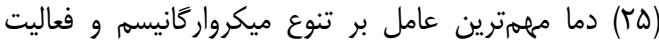

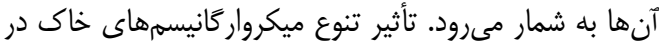

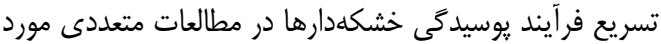

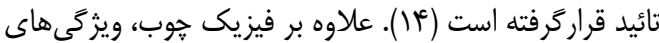

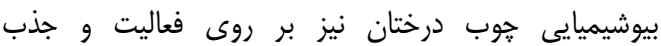

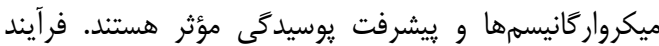

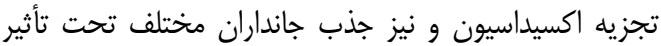

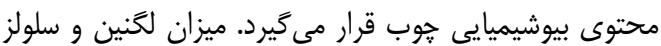

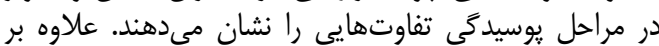

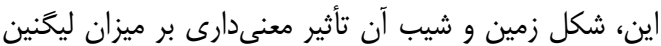

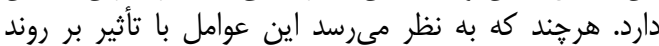

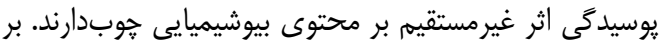

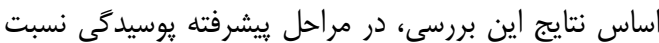

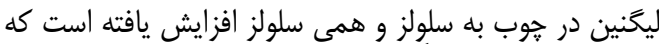
علت آن تجزيه نسبتاً كند ليحَنين در قياس با سلولز است إت 
1. Albrecht, L. 1990. Grundlagen, Ziele und Methodik der Waldökologischen Forschung in Naturwald reservaten. Bayerisches Staatsministerium für Ernährung, Landwirtschaft und Forsten, Müchen.

2. Alavi, S.J., Z.G. Amiri, M.R. Marvie Mohadjer and Z. Nouri. 2008. Spatial distribution of Ulmus glabra in relation to physiographical parameters. Journal of Environment, 43: 93-100 (In Persian).

3. Alidadi, F., M.R. Marvi-Mohadjer, V. Etemad and K. Sefidi. 2015. Decay dynamic of beech and hornbeam trees in mixed beech (Fagus orientalis lipsky) stands. Iranian Journal of Forest and Poplar Research, 22(4): 624-635 (In Persian).

4. Anonymus. 2004. Forestry plan of Asalem booklet. Forest Range and Watershed Management Organization, Iran (In Persian).

5. Barbour, M.G., J.H. Burk, W.D. Pitts, F.S. Gilliam and M.W. Schwartz. 1999. Terrestrial Plant Ecology. Benjamin/Cummings, Addison Wesley Longman, Inc. 649 pp.

6. Berg, B., B. Erhagen, M. Johansson, L. Vesterdal, M. Faituri, P. Sanborn and M. Nilsson. 2013. Manganese dynamics in decomposing needle and leaf litter - a synthesis. Canadian Journal of Forest Research, 43(12): 1127-1136.

7. Beers, T.W., P.E. Dress and L.C. Wensel. 1966. Aspect transformation in site productivity research. Journal of Forestry, 64: 691-692.

8. Bradford, M.A., R.J. Warren, P. Baldrian, T.W. Crowther, D.S. Maynard, E.E. Oldfield, W.R. Wieder, S.A. Wood and J.R. King. 2014. Climate fails to predict wood decomposition at regional scales. Nature Climate Change, 4: 625-630.

9. Bütler, R., L. Patty, R.C. Le Bayon, C. Guenat and R. Schlaepfe. 2007. Log decay of Picea abies in the Swiss Jura Mountains of Central Europe. Forest Ecology and Management, 242(2-3): 791-799.

10. Crawford, R.L. 1981. Lignin Biodegradation and Transformation. New York: John Wiley. 280pp.

11. Dunn, C.J. and J. D. Bailey. 2012. Temporal dynamics and decay of coarse wood in early seral habitats of dry-mixed conifer forests in Oregon's Eastern Cascades. Forest Ecology and Management, 276: 71-81.

12. Fukasawa, Y. 2015. The geographical gradient of pine log decomposition in Japan. Forest Ecology and Management, 349: 29-35.

13. Garrett, L.G., M.O. Kimberley, G.R. Oliver, S.H. Pearce and P.N. Beets. 2012. Decomposition of coarse woody roots and branches in managed Pinus radiata plantations in New Zealand- A time series approach. Forest Ecology and Management, 269: 116-123.

14. Garrett, L.G., G.R. Oliver, S.H. Pearce and M.R. Davis. 2008. Decomposition of Pinus radiata coarse woody debris in New Zealand. Forest Ecology and Management, 255: 3839-3845.

15. Harmon, M.E. 2009. Woody detritus mass and its contribution to carbon dynamics of old-growth forests: the temporal context. In: Wirth, C., G. Gleixner and M. Heimann (Eds.), Ecological Studies, vol. 207: Old-Growth Forests. Springer, Berlin Heidelberg, 159-190 pp.

16. Hérault, B., J. Beauchêne, F. Muller, F. WagnerBaraloto and B. BlancMartin. 2010. Modeling decay rates of dead wood in a Neotropical forest. Oe.L. ecologia, 164: 243-251.

17. Liua, W., D. Schaefer, L. Qiao and X. Liu. 2013. What controls the variability of wood-decay rates? Forest Ecology and Management, 310: 623-631.

18. Lombardi, F., P. Cherubini, R. Tognetti, C. Cocozza, B. Lasserre and M. Marchetti. 2013. Investigating biochemical processes to assess deadwood decay of beech and silver fir in Mediterranean mountain forests. Annals of Forest Science, 70(1): 101-111.

19. Mackensen, J. and J. Bauhus. 2003. Density loss and respiration rates in coarse woody debris of Pinus radiata, Eucalyptus regnans and Eucalyptus maculata. Soil Biology and Biochemistry, 35: 177-186.

20. McNab, W.H. 1993. A topographic index to quantify the effect of mesoscale landform on site productivity, Canadian Journal of Forestry Research, 23: 1100-1107

21. Müller, S.U. and N. Bartsch. 2009. Decay dynamic of coarse and fine woody debris of a beech (Fagus sylvatica L.) forest in Central Germany. European Journal of Forest Research, 128: 287-296.

22. Olson, J.S. 1963. Energy storage and the balance of producers and decomposers in ecological systems. Ecology, 44: 322-330.

23. Paletto.A. and V. Tosi. 2010. Deadwood density variation with decay class in seven tree species of the Italian Alps, Scandinavian Journal of Forest Research, 25(2): 164-173.

24. Petrillo, M., P.G. Cherubini Sartori, S. Abiven, J. Ascher, D. Bertoldi, F. Camin, A. Barbero, R. Larcher and M. Egli. 2015. Decomposition of Norway spruce and European larch coarse woody debris (CWD) in relation to different elevation and exposure in an Alpine setting. I Forest 9: 154-164.

25. Pietikaeinen, J., A.B. MariePettersson and E. Baeth. 2005. Comparison of temperature effects on soil respiration and bacterial and fungal growth rates. Microbiology Ecology, 52: 49-58.

26. Pouska, V. and M. Svoboda. 2010. The diversity of wood-decaying fungi in relation to changing site conditions in an old-growth mountain spruce forest, central Europe. European Journal of Forest Research, 129: 219-231. 
27. Russell, M.B., C.W. Woodall, A.W. D'Amato, S. Fraver and J.B. Bradford. 2014. Technical note: Linking climate change and downed woody debris decomposition across forests of the eastern United States. Bio geosciences, 11: 6417-6425.

28. Santiago, J.M. and D.R. Amanda. 2005. Dead trees as resources for Forest Wildlife. Extension fact sheet. Ohio State University Express, 12 pp.

29. Sefidi, K., D.F. Darabd and M. Sharari. 2016. The decay time and rate determination in oriental beech (Fagus orientalis Lipsky) dead trees in Asalem forests. Environmental Studies, 42(3): 551-563.

30. Sefidi, K., D.F. Esfandiary and M. Azarian. 2016. Effect of topography on tree species composition and volume of coarse woody debris in an Oriental beech (Fagus orientalis Lipsky) old growth forests, northern Iran. I Forest, 9: 658-665.

31. Sefidi, K. and V. Etemad. 2015. Dead wood characteristics influencing macro fungi species abundance and diversity in Caspian natural beech (Fagus orientalis Lipsky) forests. Forest Systems, 24(2): $1-9$.

32. Sefidi, K. 2018. Quantitative evaluation of habitat and dead tree abundance in the oriental beech (Fagus orientalis Lipsky) stands, case study from the Siahkal forests, Iranian Journal of Forest and Poplar Research, 26(3): 331-343.

33. Swanson, F.J., T.K. Kratz, N. Caine and R.G. Woodmansee. 1988. Landform Effects on Ecosystem Patterns and Processes. BioScience, 38(2): 92-98.

34. Talbot, J.M. and K.K. Treseder. 2012. Interactions among lignin, cellulose, and nitrogen drive litter chemistry-decay relationships. Ecology, 93: 345-354.

35. Taleshi, H. and M. Akbarinia. 2012. Biodiversity of Woody and Herbaceous Vegetation Species in Relation to Environmental Factors in Lowland Forests of Eastern Nowshahr. Iranian Journal of Biology, 24(5): 776-777 (In Persian).

36. Tobin, B., K. Black, L. McGurdyand and M. Nieuwenhuis. 2007. Estimates of decay rates of components of coarse woody debris in thinned Sitka spruce stands. Forestry, 80: 455-469.

37. Urbanova, M., J. Snajdr and P. Baldrian. 2015. Composition of fungal and bacterial communities in forest litter and soil is largely determined by dominant trees, Soil Biology and Biochemistry, 84: 5364.

38. Valipour, A., M. Namiranian, H. Ghazanfari, S.M. Heshmatol Vaezin, M.J. Lexer and T. Plieninger. 2014. Relationships between forest structure and tree's dimensions with physiographical factors in Armardeh forests (Northern Zagros). Iranian Journal of Forest and Poplar Research, 21(1): 30-47 (In Persian).

39. Yan, E., X. Wang, J. Huang, R. Zeng and Gong. 2007. Long - lasting legacy of forest succession and forest management: Characteristics of coarse woody debris in an evergreen broad-leaved forest of Eastern China. Forest Ecology and Management, 252: 98-107.

40. Yin, X. 1999. The decay of forest dead wood: numerical modeling and implications based on some 300 data cases from North America. Oecologia, 121: 81-98.

41. Zar, J.H. 1999. Biostatistical analysis, Ed 4. Upper Saddle River, NJ: Prentice Hall. 


\title{
The Influence of Geomorphological Characteristics of Forest Sites on the Decay Dynamics of Dead Trees in Asalem Forests, Western Hyrcanian Region
}

\author{
Kiomars Sefidi \\ Associate Professor, Faculty of Agriculture and Natural Resources of Mohaghegh Ardabili, Ardebil, Iran \\ (Corresponding Author: Kiomarssefidi@gmail.com) \\ Receive: August 29, $2018 \quad$ Accepted: December 2, 2018
}

\begin{abstract}
Knowledge of the decay trend of dead trees and site factors affecting their functions, plays an important role in the development of conservation management plans in forestry projects. This research was conducted in Asalem beech forests in northern Iran to assess the impact of physiographic features of the site on the process of dead trees decay. A total of 90 sample cuts of dead beech stumps were randomly selected and studied. For each sample, species, diameter and decay classes were recorded. The effect of aspect Slope Index (ASI), Terrain Shape Index (TSI) and Landform Index (LI) on the physical properties of dead wood (density, weight, moisture content) and biochemical components of wood (lignin, cellulose and hemicellulose) on the process of decay were investigated. The mean decay rate of dead beech stumps calculated 0.061 and the mean of beech was 0.32 that varied between 0.05 and 0.62 grams per cubic meters. The results revealed decay process requires about 46 years and the four classes decay showed significant difference in terms of wood density, lignin and cellulose. Meanwhile, the LI was the most important physiographic factors influencing the decay rate of dead trees. The lignin content of the deadwoods was significantly influenced by the form of land. Accordingly, it can be concluded that maintenance certain volume of dead tree in the forest stand structure need to taking account into the dynamics of dead trees, as well as the effects of geomorphological characteristics of site, especially land form conditions
\end{abstract}

Keywords: Decay Constant, Decay Grade, Landform Index (LI), Physiography, Terrain Shape Index (TSI) 